\title{
Uncertainty Analysis in the Measurement of Switching Losses in GaN FETs Power Converters
}

\author{
Marco A. Azpúrua \\ Universitat Politècnica de Catalunya \\ Barcelona, Spain \\ email: marco.azpurua@upc.edu
}

\author{
Marc Pous \\ Universitat Politècnica de Catalunya \\ Barcelona, Spain \\ email: marc.pous@upc.edu
}

\author{
Ferran Silva \\ Universitat Politècnica de Catalunya \\ Barcelona, Spain \\ email: ferran.silva@upc.edu
}

\begin{abstract}
This paper analyses the method for measuring the switching losses in Gallium Nitride (GaN) Field Effect Transistors (FETs) employed in highly efficient power electronics applications, particularly in DC-DC converters. In this regard, the switching losses are measured through the integration of the product of the voltage multiplied by the current during the turn-on and turn-off intervals of the switching process. The objective of this analysis is to identify, model, correct/minimize and quantify the main contributions to the switching losses' measurement uncertainty following a topdown approach. First, a set of general specifications for a suitable test bench is given. Subsequently, the distortion of the oscilloscope-probe system and the influence of the current shunt is studied along with a method for correcting the waveform distortion. Then, based on the model describing the general measurement method, the individual sources of uncertainty are stripped down, giving guidance regarding how to estimate each of them. Finally, such individual contributions to the switching losses measurement uncertainty are propagated and combined following the guidelines of the "Guide to the Expression of Uncertainty in Measurement".
\end{abstract}

Keywords-GaN FETs, measurement uncertainty, power converters, switching loss, waveform measurements.

\section{INTRODUCTION}

Gallium Nitride $(\mathrm{GaN})$ is a high-speed wide bandgap semiconductor that is increasingly being employed in transistor manufacturing. GaN power transistors for switching and linear applications have brought benefits such as higher efficiency, faster response, and smaller size in comparison with their silicon counterparts [1]. The switching speed of low voltage GaN switches can be two orders of magnitude superior compared to a typical silicon MOSFET [2]. Likewise, the on-state resistance/breakdown voltage tradeoff of $\mathrm{GaN}$ FETs has been reported to be three orders of magnitude better than Si-based power switching technology [3].

Nowadays, there is widespread use of $\mathrm{GaN}$ devices in diverse power converter applications. In general, power converters contain several switching devices and the largest contribution to the power converter losses comes from them. In this regard, conduction loss depends on the on-state resistance $\left(R_{o n}\right)$, while the switching loss occurs at the turn-on and turn-off of the devices [4]. When compared to $\mathrm{Si}$ MOSFETs devices, GaN switches are characterized by lower $R_{o n}$ and by a lower capacitance, which means they allow for higher switching frequencies. Accordingly, they are an excellent alternative for designing more efficient power converters.
Quantifying the power losses of a power converter is a fundamental part of their design process. In that sense, one approach to estimate the power losses is to rely on the information given by the manufacturer in the switching device datasheet. Alternatively, a designer could use a SPICE model, if it is available [4]. Nonetheless, both options are not generally viable. Commonly, the datasheets of GaN switching devices do not specify switching losses and validated SPICE models are not widely accessible, even if remarkable progress has been made in that direction [5]-[7]. In consequence, measurements are still the only feasible alternative for characterizing the switching loss in many GaN devices.

Switching loss is often measured by integrating the product of the voltage across the switch and the current flowing through it over the switching time window. This timedomain approach requires acquiring the voltage and current waveforms by using a suitable oscilloscope with a set of calibrated and compensated probes with sufficient bandwidth [8]. Even if the measurement method is conceptually straightforward, several measurement challenges emerge from the sharp switching transitions that can be reached by $\mathrm{GaN}$ devices [2], with rise times in the nanosecond range.

Fidelity in the V/I waveform measurements is fundamental for the accurate evaluation of the switching losses and, therefore, oscilloscope probes have a major role to play in it [9]. Previous research has investigated the complex influence of the probe-oscilloscope system in the measured current and voltage waveforms. Particularly, in [10] the impact of the delay, distortion, and overshoots effects in the switching losses measurement error was studied. Likewise, inverse probe models for correcting the previously mentioned sources of error have been proposed [11].

Provided the appropriate measurement system and the application of the corresponding waveform distortion correction methods, the next step is to estimate the measurement uncertainty in the switching losses assessment. This is important for reporting reliable switching loss specifications for the device under assessment and for understanding the most error sensitive variables.

In this regard, this paper presents a top-down analysis for identifying, modeling, correcting/minimizing, and quantifying the main contributions to the uncertainty in the switching losses' measurement. Then, the uncertainty sources are propagated according to a model for the switching loss measurement method following the procedure of the "Guide to the Expression of Uncertainty in Measurement" [12]. 


\section{Measurement of Losses In Switching Devices}

The instantaneous value of the power losses, $p(t)$, in a switching device is given by the product of the drain-source voltage, $v_{D S}(t)$ and the drain current, $i_{D}(t)$. However, due to the periodic nature of the switching process, it is usually convenient to express the losses as an average value over a switching period, $T$, that is,

$$
P_{\text {ave }}=\frac{1}{T} \int_{t_{0}}^{t_{0}+T} p(t) d t=\frac{1}{T} \int_{t_{0}}^{t_{0}+T} v_{D S}(t) i_{D}(t) d t
$$

Fig. 1 represents the time-dependent behavior of the power loss in the switching device. Here, it is possible to identify three regions, the turn-on, and turn-off regions, and the conduction region. On the one hand, switching losses ( $E_{\text {on }}$ and $\left.E_{o f f}\right)$ occur in the turn-on and turn-off regions due to the switch parasitic capacitance on the gate that limits the speed of the switching device. On the other hand, some power is dissipated during the conduction state, $E_{\text {cond }}$. This conduction loss depends on the voltage drop across the on-state switch resistance.

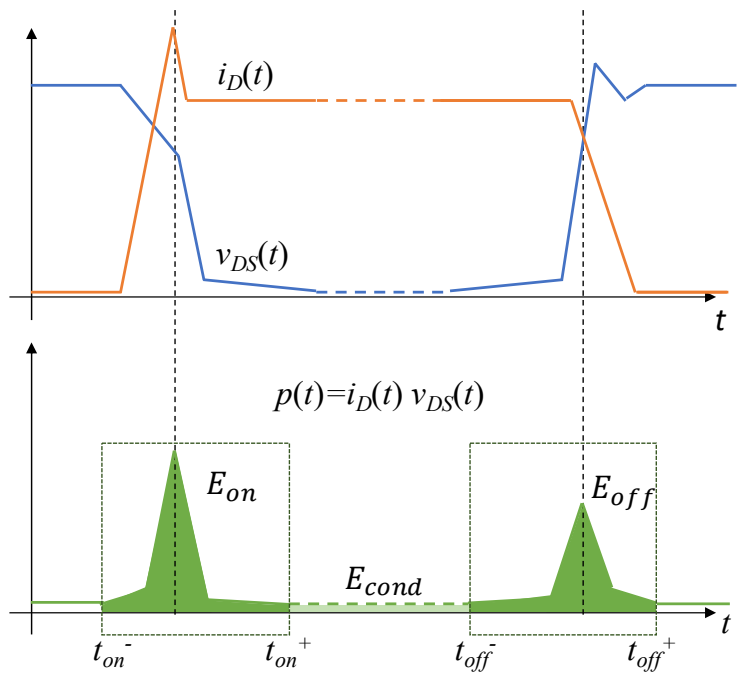

Fig. 1. Switching loss measurement through the product of the drain-source voltage and the drain current waveforms.

For $\mathrm{GaN}$ switches $R_{o n}$ is in the range of hundreds of milliohms, which translates to smaller conduction loss in comparison with the switching loss. In fact, switching loss is the majority contribution to the total energy losses in $\mathrm{GaN}$ transistors operating in hard-switching applications, between $30 \%$ and $40 \%$ [13].

In that sense, the total switching energy loss, $E_{S W}$, is defined as

$$
E_{S W}=E_{o n}+E_{o f f}=\int_{t_{o n^{-}}}^{t_{o n^{+}}} p(t) d t+\int_{t_{o f f^{-}}}^{t_{o f f^{+}}} p(t) d t
$$

where $\left[t_{o n}{ }^{-}, t_{o n}{ }^{+}\right]$and $\left[t_{o f f}, t_{o f f}{ }^{+}\right]$time intervals. It is important to notice the switching time interval end when the waveform oscillations have been completely damped [14]

Consequently, the measurement of losses in switching devices requires synchronous measurement of $v_{D S}(t)$ and $i_{D}(t)$. In principle, this is performed with an oscilloscope as shown in Fig. 2. In this regard, the voltage waveforms can be sensed directly by using passive and/or differential probes while the current waveforms are measured through resistive shunts.

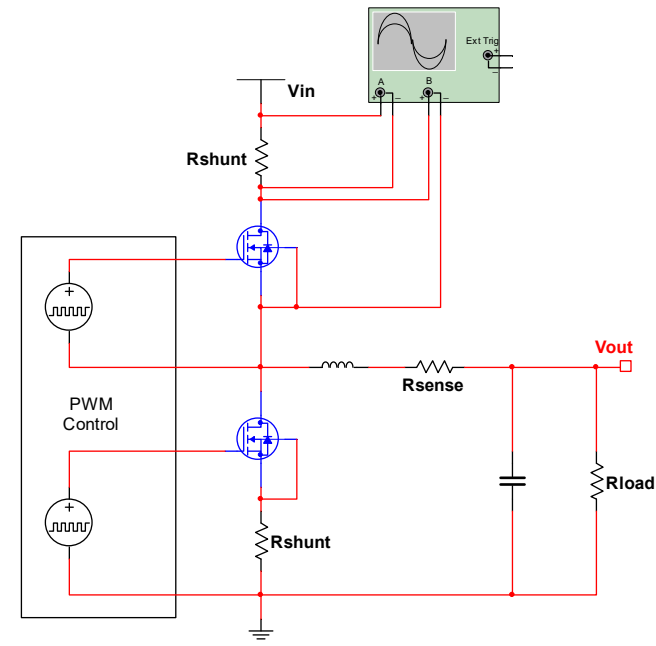

Fig. 2. Measurement of switching waveforms in the high side of a generic DC-DC Half-bridge buck converter.

However, since the switching process in $\mathrm{GaN}$ devices is characterized by waveforms with rise time nanosecond range, the measurement of the switching waveforms is sensitive to timing, offset, and distortion errors. Therefore, voltage and current waveform measurements must be carried very carefully, considering the following best practices.

\section{GENERAL RECOMMENDATIONS FOR MAKING HiGH-SPEED SWITCHING WAVEFORM MEASUREMENTS}

In the first place, an appropriate measurement setup must be used to accurately measure high-speed switching waveforms. For instance, for measuring switching voltage waveforms, the combined rise time of the oscilloscope, $t_{s}$, and of the passive/differential probe, $t_{p}$, must be small with respect to the actual $10 \%-90 \%$ edge time, $t_{e}$, of the voltage waveform to be measured [2]. In other words, this means,

$$
t_{\text {meas }}=\sqrt{t_{s}^{2}+t_{p}^{2}+t_{e}^{2}} \leq(1+\Delta) t_{e},
$$

where $\Delta$ is the maximum timing error allowable according to the required measurement timing accuracy. The combined $3 \mathrm{~dB}$ bandwidth of the oscilloscope-probe system must satisfy the condition in (4), that is,

$$
B W_{3 d B} \geq \frac{0.35}{\sqrt{t_{s}^{2}+t_{p}^{2}}}=\frac{0.35}{(\sqrt{2+\Delta} \sqrt{\Delta}) t_{e}} .
$$

For example, if $\Delta \leq 1 \%$ is the target accuracy for the timing and the edge rise times to be measured are in the nanosecond and sub-nanosecond range ( $5 \mathrm{~ns}-500 \mathrm{ps})$, then the measurement system bandwidth must range between $500 \mathrm{MHz}$ and $5 \mathrm{GHz}$. For instance, using a measurement system with a bandwidth of $1 \mathrm{GHz}$ for both the probe and the oscilloscope would be enough in the first case but, conversely, would cause an unacceptable timing error of more than $20 \%$ in the more stringent scenario.

On the other hand, measuring the current waveform is challenging because oscilloscope current probes (hall effect and Rogowski probes) have shortcomings for this application. In this regard, current probes are too large for fitting in the power converter, and even if it were possible to do it, the insertion impedance of the current probe would be significant compared to the circuit, thus affecting the measurement accuracy. Therefore, in practice, current probes are not 
suitable for this purpose. With oscilloscope current probes discarded, sensing resistors such as coaxial shunts and planar resistors are a reasonable alternative for transforming the current waveform into a voltage waveform to be measured [2].

In addition to the previous considerations, a set of best practices must be followed to ensure accurate switching loss measurements. The most important ones are [8]:

- Offset errors. Differential voltage probes can have a DC offset due to their internal amplifiers. With the differential probe inputs shorted and no input signal applied such offset must be corrected to be zero.

- Timing errors. The current and voltage waveform experience different propagation delay. This means the inter-channel timing must be adjusted to de-skew the oscilloscope channels.

- Dynamic range and noise reduction. The signal-tonoise ratio must be optimized by keeping the waveform amplitude as large as possible without, of course, saturating the channel input. Likewise, it is advisable to combine waveform averaging and highresolution acquisition modes to reduce the uncorrelated random noise.

- Probe loading, input capacitance and, ground inductance. High-impedance probes above $1 \mathrm{GHz}$ are not available and the actual input impedance of the probe significantly decreases with frequency. Differential probes usually have lower capacitances than passive probes, but they usually have a limited dynamic range. Using the appropriate prove tip and grounding can reduce the ground loop inductance [15].

Following the above-mentioned recommendations and using an appropriate test bench is necessary, but not sufficient, for ensuring accurate measurement of the switching losses. This is because the probe-oscilloscope system introduces distortion in the waveform, particularly when the probe's input capacitance is of the same order of magnitude as the capacitance of the DUT [16]. The next section studies the distortion of the probe-oscilloscope system through the circuit modeling and simulation approach.

\section{WAVEFORM DISTORTION CORRECTION}

As the bandwidth of the switching waveforms increases, the parasitic effects that limit the response of the probeoscilloscope system emerge and the waveform distortion produced by them can significantly alter the measurements.

In line with the above, let $x(t)$ be an input signal, and $y(t)$ the corresponding measured (output) waveform, then

$$
y(t)=p(t) * x(t)+n(t)
$$

where $p(t)$ is the probe-oscilloscope system impulse response and $n(t)$ is the noise in the measurements. However, if it is assumed the probe-oscilloscope system is linear timeinvariant (LTI) and that the noise is neglectable, then the complex value transfer function of the probe-oscilloscope system can be expressed as the frequency response, $P(j \omega)$,

$$
P(j \omega)=|P(j \omega)| \angle \varphi(j \omega)=\frac{Y(j \omega)}{X(j \omega)}
$$

where $X(j \omega)$ and $Y(j \omega)$ are the Fourier transform of system input and output, respectively.
Therefore, if the probe-oscilloscope system frequency response is known and if the Fourier transform exists for $x(t)$ and $y(t)$, the amplitude and phase distortion caused by the measurement process can be corrected.

Now, let $y(t)$ to be periodic with period $T$, that is, $y(t)=y(t+T)$. In consequence, $y(t)$ can be written a Fourier series,

$$
y(t)=c_{0}+\sum_{n=1}^{\infty}\left|c_{n}\right| \cos \left(n \omega_{0} t+\theta_{n}\right)
$$

where $\omega_{0}=2 \pi / T$. The constant coefficient in (5), $c_{0}$, is the average amplitude of the waveform over one period of time [17], that is

$$
c_{0}=\frac{1}{T} \int_{0}^{T} y(t) d t
$$

whereas magnitude and phase of the harmonic components of the Fourier series are given by

$$
c_{n}=\left|c_{n}\right| e^{j \theta_{n}}=\frac{2}{T} \int_{0}^{T} y(t) e^{-j n \omega_{0} t} d t .
$$

Consequently, in steady-state, $x(t)$ can be rewritten as the superposition of sinewaves in (8),

$$
x(t)=\frac{c_{0}}{P_{0}}+\sum_{n=1}^{\infty} \frac{\left|c_{n}\right|}{P_{n}} \cos \left(\omega_{n} t+\theta_{n}-\varphi_{n}\right)
$$

where $\omega_{n}=n \omega_{0}$ is the angular frequency of the $n$-th harmonic component and $P_{n} \angle \varphi_{n}$ is the phasor obtained by evaluating $P(j \omega)$ at $\omega_{n}$ for $n=1,2,3 \ldots$

In practice, the number of harmonic components in (8) can be limited according to the measurement system bandwidth in order to avoid the non-realizable infinite summation. Thus, if $B$ is the bandwidth of the measurement system then the order of the highest frequency harmonic, $N$, would be given by,

$$
N=B T \text {. }
$$

\section{THE TRANSFER FunCtion OF THE PROBE-OSCILLOSCOPE SYSTEM}

The transfer function of the probe-oscilloscope system can be extracted from circuit models. Circuit models are particularly helpful because they are easy to simulate and help to understand the effect of the parasitic elements in the frequency response of the measurement system. For providing realistic correction factors, the probe-oscilloscope system models must be fitted and validated with regards to calibration data and the instrument specifications.

Within the scope of this paper, passive voltage probes are used for measuring voltage waveforms while coaxial current shunts are used for measuring current waveforms. In what follows both types of transducers will be analyzed.

\section{A. The frequency response of passive voltage probes}

Fig. 3 shows a probe-oscilloscope circuit model for a generic passive voltage probe. In this model, the tip, the cable, the compensation circuit, and the oscilloscope impedance are considered since they have a direct influence on the measured voltage. 


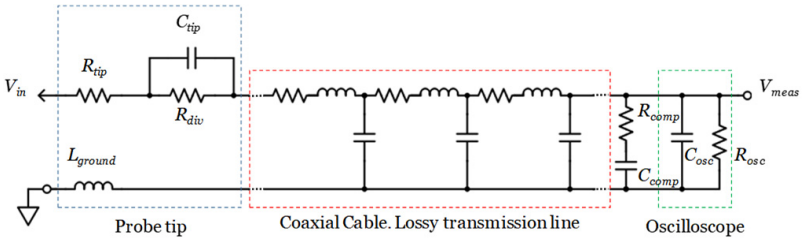

Fig. 3. Probe-oscilloscope model for a generic passive voltage probe.

As a particular example, let us tune the lumped and distributed parameters (resistances, capacitances, and inductances) in the model above according to the specified frequency response of the measurement system formed by the passive voltage probe TPP1000 from Tektronix and the Digital Real-Time sampling Oscilloscope (DRTO) DPO5104B from the same manufacturer [18], [19].

On the one hand, $R_{\text {osc }}$ is assumed to be $1 \mathrm{M} \Omega$ as stated in the oscilloscope specifications for high impedance measurements and $R_{d i v}$ will depend on the attenuation factor, which means that for a DC voltage division factor of $10, R_{d i v}$ must be $9 \mathrm{M} \Omega$. It is also specified that both elements have a $3 \mathrm{~dB}$-bandwidth of $1 \mathrm{GHz}$. Accordingly, the theoretical rise time $(10 \%-90 \%)$ of the oscilloscope is 350 ps at full bandwidth. In such conditions, the oscilloscope input impedance is set to $50 \Omega$ and $C_{o s c}$ is given by,

$$
C_{\text {osc }}=\frac{t_{10 \%-90 \%}}{Z_{\text {osc }} \times \ln \left(\frac{90 \%}{10 \%}\right)} \approx 3.1 \mathrm{pF}
$$

Moreover, in [15] it is reported that $L_{\text {loop }}$ is $10 \mathrm{nH}$ if the $1 / 2$ " ground spring is used. From the TPP1000 specifications, it is known that its cable is $1.3 \mathrm{~m} \pm 3 \mathrm{~cm}$ long. Finally, from the probe's instructions we now that its typical input impedance behaves as shown in Fig. 4 [18].

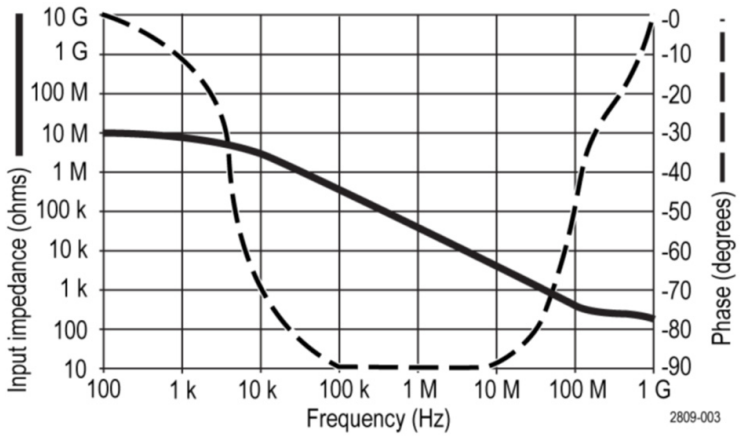

Fig. 4. Input impedance as a function of the frequency for the passive voltage probe TPP1000 from Tektronix [18].

Therefore, the values for the lumped and distributed elements shown in Fig. 5 have been selected and tuned for satisfying the before mentioned conditions.

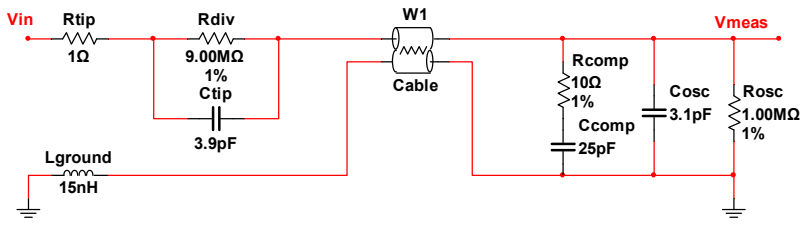

Fig. 5. SPICE model for the passive voltage probe TPP1000 from Tektronix.

After performing an AC sweep analysis with NI Multisim 14.2, the results of the input impedance of the model are in excellent agreement with the TPP1000 specifications as shown in Fig. 6. Consequently, the probe simulation model has been validated and it is reliable for estimating the probe transfer function through simulation.

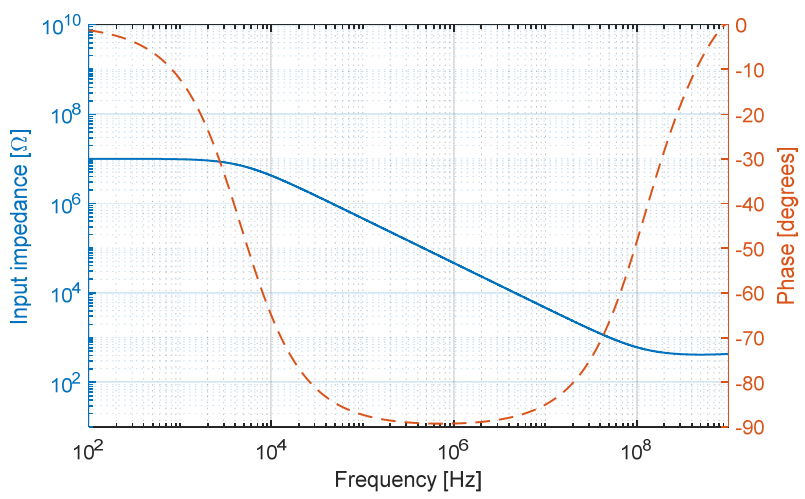

Fig. 6. Input impedance as a function of the frequency for the SPICE model of the TPP1000.

Hence, the simulated frequency response of the TPP1000DPO5104B probe-oscilloscope system is shown in Fig. 7. It is verified that the proposed model $3 \mathrm{~dB}$ cutoff frequency is approximately $1 \mathrm{GHz}$. Likewise, the flat frequency response exhibited by the probe in both magnitude and phase up to $100 \mathrm{MHz}$ indicates that the probe-oscilloscope distortion might be neglected without compromising significantly the measurement's accuracy for waveforms with spectral content limited to $100 \mathrm{MHz}$. Conversely, it is evident that amplitude and phase distortion correction is mandatory when measuring high-speed switching waveforms.

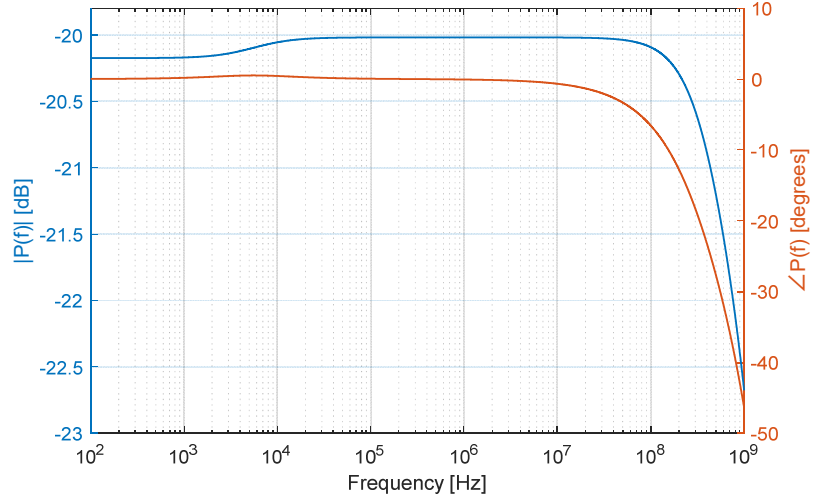

Fig. 7. The simulated transfer function of the TPP1000-DPO5104B probeoscilloscope system.

\section{B. The frequency response of coaxial current shunts}

Basically, a shunt is a resistor across which a voltage is measured for sensing the current flowing through it. Hence, the shunt resistance in direct current (dc) conditions, $R_{\text {shunt }}$, is usually specified as the voltage-current conversion factor. For power electronics applications suitable $R_{\text {shunt }}$ values are, typically, in the range of milliohms. In general, keeping $R_{\text {shunt }}$ low allows reducing the impact of the shunt voltage drop in the circuits and minimizes the power dissipated in the shunt, thus reducing thermally induced resistance drift.

On the other hand, for alternating current and high-speed current waveform measurements (broadband frequency content), it is necessary to have characterized the frequency response of the shunt impedance, $Z_{\text {shunt }}(f)$. Shunts have an intrinsic inductance, which is influenced by its design 
structure [20]. The shunt inductance, $L_{\text {shunt }}$, is the key parameter limiting its frequency response. Considering a simple series RL circuit mode, $Z_{\text {shunt }}(j \omega)=R_{\text {shunt }}+$ $j \omega L_{\text {shunt }}$, the shunt's $3 \mathrm{~dB}$ cutoff frequency, $f_{c}$, is the frequency at which $R_{\text {shunt }}$ is equal to the shunt's reactance,

$$
f_{c}=\frac{R_{\text {shunt }}}{2 \pi L_{\text {shunt }}} \text {. }
$$

Bearing in mind we require measuring the switching waveforms of GaN devices, $f_{c}$ must be at least equal to the bandwidth of the rest of the measurement system. This requires the shunt inductance to be in the femtohenry range, that is, almost nulling the magnetic flux enclosed by the measuring loop.

In that sense, coaxial shunts, formed by two coaxial tubes that carry current in opposite directions, cancel the magnetic field inside the inner hollow tube. This inner tube is made of resistive material and when the leads are attached to the inner surface no flux linkage limits the shunt response. However, in this case, the shunt time response is determined by the propagation velocity of the signal through the shunt foil and the thickness of the sheet metal of the inner tube. The delay in response due to skin effect increases with tube thickness and conductivity. It follows that, for a fast response, a thin tube of highly resistive material is required. [21].

Therefore, the impedance characteristic of the coaxial current shunt is primarily flat from DC to the cutoff frequency (11). Hence, the equivalent $L_{\text {shunt }}$ of the series RL circuit model can be calculated from its rise time. Experimentally, the shunt rise time $(10 \%-90 \%)$ can be calibrated using a step function of current produced by a coaxial line pulse generator. In consequence,

$$
L_{\text {shunt }}=\frac{R_{\text {shunt }} \cdot t_{10 \%-90 \%}}{\operatorname{Ln}(9)} .
$$

As an example, let us examine the coaxial shunt SDN-41405 from T\&M Research Products, Inc. Its nominal resistance is $50 \mathrm{~m} \Omega$ and its specified rise time is $0.18 \mathrm{~ns}$. The rise time has been calibrated using a reference pulse with $\mathrm{di} / \mathrm{dt}>10^{12} \mathrm{~A} / \mathrm{s}$. According to (12), this means $L_{\text {shunt }} \approx 4,1 \mathrm{fH}$ and $f_{c} \approx 2 \mathrm{GHz}$. To the best of authors' knowledge, nowadays, only SDN-414 series from T\&M Research Products, Inc. offers a suitable performance for GaN switching waveform measurements.

\section{UNCERTAINTY IN THE MEASUREMENT OF SWITCHING LOSS}

The analysis of the measurement uncertainty in the switching loss measurement is made upon the analytic description of the measurement model using a top-down approach. Then, individual sources of uncertainty are propagated and combined flowing the recommendations in the GUM.

Accordingly, the measurement uncertainty in the $E_{S W}, u_{S W}$, is the combination of the measurement uncertainties of the switching losses during the on and off transitions, $u_{O N}$ and $u_{\mathrm{OFF}}$, respectively. In other words,

$$
u_{S W}=\sqrt{\left(u_{O N}\right)^{2}+\left(u_{O F F}\right)^{2}} .
$$

The energy losses in (2) are calculated by integrating the instantaneous power loss during the switching time intervals.
In this regard, the measured power loss is a time-discrete vector calculated as the product of the sampled voltage and current waveforms. This means, the energy loss in the time interval $\left[t_{1} ; t_{2}\right], E_{\left[t_{1} ; t_{2}\right]}$, is estimated by approximating the power integral as a Riemann sum, that is,

$$
E_{\left[t_{1} ; t_{2}\right]} \approx T_{s} \sum_{n=n_{1}}^{n_{2}} p\left[n T_{s}\right]
$$

where $T_{S}$ is the sampling interval of the waveforms and $n=n_{1}, n_{1}+1, \ldots, n_{2}$ is the number of the $n$-th sample.

However, considering the timing error during the transition of the switching events, $\Delta$, given as a percentage of the waveforms edge time (either rise/fall times), $t_{\mathrm{e}}$, then the integration interval $\left[t_{1} ; t_{2}\right]$ is also uncertain. Consequently, the error in the energy loss due to an uncertain time interval, $E_{\Delta}$, can be bounded as,

$$
E_{\left[t_{1}+\Delta t_{e} ; t_{2}-\Delta t_{e}\right]} \leq E_{\Delta} \leq E_{\left[t_{1}-\Delta t_{e} ; t_{2}+\Delta t_{e}\right]} .
$$

With no further knowledge about the distribution of $E_{\Delta}$, a rectangular distribution is assumed and the uncertainty contribution of $E_{\Delta}, u_{E_{\Delta}}$, is given as

$$
u_{E_{\Delta}}=\frac{E_{\left[t_{1}-\Delta t_{e} ; t_{2}+\Delta t_{e}\right]}-E_{\left[t_{1}+\Delta t_{e} ; t_{2}-\Delta t_{e}\right]}}{\sqrt{3}} .
$$

Assuming independent of the variables in (12), and using the first-order uncertainty propagation rule [12], the uncertainty in $E_{\left[t_{1} ; t_{2}\right]}, u_{E_{\left[t_{1} ; t_{2}\right]}}$, can be expressed as,

$$
u_{E_{\left[t_{1} ; t_{2}\right]}}=\sqrt{\left(\frac{E_{\left[t_{1} ; t_{2}\right]}}{T_{s}} u_{T_{s}}\right)^{2}+T_{s}{ }^{2} \sum_{n=n_{1}}^{n_{2}} u_{p, n^{2}}{ }^{2} u_{E_{\Delta}}{ }^{2}}
$$

where $u_{T_{S}}$ is the uncertainty of the sampling interval and $u_{p, n}$ is the uncertainty in the measurement of the instantaneous power loss of the $n$-th sample. Consequently, $u_{O N}=$

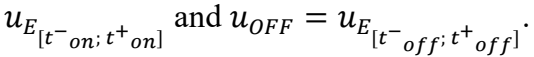

In this regard, the absolute error of the sampling interval, $e_{T_{S}}$, is usually expressed as

$$
e_{T_{S}}= \pm K T_{S}
$$

where $K$ is a fixed constant value that represents the combined relative error of caused by the elements of the oscilloscope acquisition system, namely, vertical noise, nonlinearity, digitizing errors, interleaving errors, aperture uncertainty (sample time jitter), interpolation uncertainty and round-off errors. Therefore, assuming a uniform distribution for $e_{T_{S}}$, the standard uncertainty in the sampling interval is,

$$
u_{T_{S}}=\frac{2 e_{T_{S}}}{\sqrt{3}} .
$$

Moreover, the $u_{p, n}$ corresponds to the combination of the uncertainty contributions in the voltage, $u_{v}$, and current, $u_{i}$, waveform points, that is,

$$
u_{p, n}=\sqrt{\left(i_{n} u_{v}\right)^{2}+\left(v_{n} u_{i, n}\right)^{2}} \text {. }
$$

where $i_{n}$ and $v_{n}$ are the instantaneous value of the current and voltage at the $n$-th waveform point. With respect $u_{v}$, it is quantified as the combination of the sources of uncertainty that limit the accuracy of the oscilloscope vertical system, and it can be expressed as, 


$$
u_{v}=\sqrt{\left(u_{A D C}\right)^{2}+\left(u_{\text {noise }}\right)^{2}+\left(u_{o f f}\right)^{2}+\left(u_{\text {probe }}\right)^{2}}
$$

where $u_{A D C}, u_{\text {noise }}, u_{o f f}$, and $u_{\text {probe }}$ are the uncertainty contributions due to the ADC quantization error, the random noise in the waveforms, the offset error and the combined error due to the oscilloscope probing effects.

On the other hand, the current waveform is measured indirectly through the shunt voltage and using Ohm's law, that is,

$$
i(t)=\frac{v_{\text {shunt }}(t)}{Z_{\text {shunt }}}
$$

Therefore, the measurement uncertainty for the $n$-th current waveform point, $u_{i, n}$, is given by,

$$
u_{i, n}=\sqrt{\left[\left(\frac{u_{v_{\text {shunt }}}}{Z_{\text {shunt }}}\right)^{2}+\left(\frac{v_{\text {shunt }, n}}{\left(Z_{\text {shunt }}\right)^{2}} u_{Z_{\text {shunt }}}\right)^{2}\right]}
$$

where $Z_{\text {shunt }}$ is the nominal value of the shunt impedance, $v_{\text {shunt, } n}$ is the $n$-th shunt voltage waveform point and $u_{Z_{\text {shunt }}}$ is the uncertainty in the value of $Z_{\text {shunt }}$. The assigned value and distribution for $u_{Z_{\text {shunt }}}$ will depend on the information available on the transducer, i.e. a calibration certificate or a tolerance specification. Finally, the uncertainty in the shunt voltage waveforms points is calculated using the same criterion that in (20).

It is important to highlight that to estimate the uncertainty above, the oscilloscope's specifications must be accounted for in terms of its $\mathrm{ADC}$ resolution and the effective number of bits, offset error, gain error, input impedance, etc. In fact, there is no single set of standard figures for defining the accuracy of the oscilloscope vertical system in dynamic conditions. In consequence, from this point onwards the uncertainty analysis must be made based on the information available for a particular implementation of a test bench.

Finally, it is important to remark the uncertainty analysis presented so far assumes many plausible systematic errors have been mitigated by following the best practices presented in section III and that the frequency-dependent waveform distortion has been corrected using the method exposed in section IV.

\section{DISCUSSION}

Through the analysis performed, it has been highlighted how challenging it is to conduct accurate measurements of switching losses in the case of GaN FETs hard switching applications. In fact, what may have been regarded as a remarkably simple test procedure for preceding transistor technologies requires considering several factors that were previously neglected. The most significant one is the probeoscilloscope system distortion.

Having a detailed analysis of the measurement uncertainty allow realizing the limitations a test bench has for the characterization of the switching losses of a given GaN FET, thus allowing a rational decision regarding if this is enough for the intended application.

A follow-up paper is expected to cover the analysis of a particular test bench, delivering experimental results, the evaluation of the uncertainty and the validation of the numerical results using the Monte Carlo method.

\section{ACKNOWLEDGMENT}

This research work is carried in the framework of the ADVENT project (Grant Number: 16ENG06 ADVENT) which is supported by the European Metrology Programme for Innovation and Research (EMPIR). The EMPIR initiative is co-funded by the European's Horizon 2020 research and innovation programme and the EMPIR Participating States.

\section{REFERENCES}

[1] T. J. Flack, B. N. Pushpakaran, and S. B. Bayne, "GaN Technology for Power Electronic Applications: A Review," J. Electron. Mater., vol. 45, no. 6, pp. 2673-2682, Jun. 2016.

[2] S. Sandler, "Faster-Switching GaN : Presenting a number of interesting measurement challenges," IEEE Power Electron. Mag., vol. 2, no. 2, pp. 24-31, 2015.

[3] D. Jin and J. A. Del Alamo, "Methodology for the study of dynamic ON-resistance in high-voltage GaN field-effect transistors," IEEE Trans. Electron Devices, 2013.

[4] M. Okamoto, G. Toyoda, E. Hiraki, T. Tanaka, T. Hashizume, and T. Kachi, "Loss evaluation of an AC-AC direct converter with a new GaN HEMT SPICE model," in IEEE Energy Conversion Congress and Exposition: Energy Conversion Innovation for a Clean Energy Future, ECCE 2011, Proceedings, 2011.

[5] L. Wu and M. Saeedifard, "A Simple Behavioral Electro-Thermal Model of GaN FETs for SPICE Circuit Simulation," IEEE J. Emerg. Sel. Top. Power Electron., 2016.

[6] S. Khandelwal et al., "ASM GaN: Industry Standard Model for GaN RF and Power Devices--Part 1: DC, CV, and RF Model," IEEE Trans. Electron Devices, pp. 1-7, 2018.

[7] S. A. Albahrani, D. Mahajan, J. Hodges, Y. S. Chauhan, and S. Khandelwal, "ASM GaN: Industry Standard Model for GaN RF and Power Devices--Part-II: Modeling of Charge Trapping," IEEE Trans. Electron Devices, pp. 1-8, 2018.

[8] Tektronix, "Measuring Power Supply Switching Loss with an Oscilloscope," 2016.

[9] K. Ammous et al., "Error in estimation of power switching losses based on electrical measurements," in 2000 IEEE 31st Annual Power Electronics Specialists Conference. Conference Proceedings (Cat. No.00CH37018), 2000, vol. 1, pp. 286-291 vol.1.

[10] K. Ammous, H. Morel, and A. Ammous, "Analysis of Power Switching Losses Accounting Probe Modeling," IEEE Trans. Instrum. Meas., vol. 59, no. 12, pp. 3218-3226, 2010.

[11] K. Ammous, H. Morel, and A. Ammous, "Inverse Models of Voltage and Current Probes," IEEE Trans. Instrum. Meas., vol. 60, no. 12, pp. 3898-3906, 2011.

[12] Joint Committee For Guides In, "Evaluation of measurement data Guide to the expression of uncertainty in measurement," Int. Organ. Stand. Geneva ISBN, 2008.

[13] A. Lidow, J. Strydom, M. de Rooij, and D. Reusch, GaN Transistors for Efficient Power Conversion: Second Edition. 2014.

[14] K. Wang, M. Tian, H. Li, F. Zhang, X. Yang, and L. Wang, "An improved switching loss model for a $650 \mathrm{~V}$ enhancement-mode GaN transistor," in 2016 IEEE 2nd Annual Southern Power Electronics Conference, SPEC 2016, 2016.

[15] S. Biswas, D. Reusch, M. de Rooij, and T. Neville, "Evaluation of measurement techniques for high-speed GaN transistors," in 2017 IEEE 5th Workshop on Wide Bandgap Power Devices and Applications (WiPDA), 2017, pp. 105-110.

[16] C. Xiao, G. Chen, and W. G. H. Odendaal, "Overview of Power Loss Measurement Techniques in Power Electronics Systems," IEEE Trans. Ind. Appl., vol. 43, no. 3, pp. 657-664, May 2007.

[17] C. R. Paul, Introduction to Electromagnetic Compatibility: Second Edition. 2006.

[18] Tektronix, "TPP1000. 1 GHz 10X Passive Probe. Instructions.”.

[19] Tektronix, "MSO5000/DPO5000 Series Mixed Signal Oscilloscopes Datasheet," 2018.

[20] J. A. Ferreira, W. A. Cronje, and W. A. Relihan, "Integration of high frequency current shunts in power electronic circuits," IEEE Trans. Power Electron., vol. 10, no. 1, pp. 32-37, 1995.

[21] B. Lago and R. Eatock, "Coaxial shunt," Proc. Inst. Electr. Eng., vol. 114, no. 9, pp. 1317-1324, 1967. 